\title{
Near-horizon symmetries of the Schwarzschild black holes with supertranslation field
}

\author{
Mikhail Z. Iofa \\ Skobeltsyn Institute of Nuclear Physics, Lomonosov Moscow State University, Moscow 119992, Russia
}

(Received 15 November 2018; published 29 March 2019)

\begin{abstract}
Using the exact solution to Einstein equations of Compere and Long for the Schwarzschild metric containing the supertranslation field, we study the near-horizon symmetries of the metric. We consider a class of metrics with a supertranslation field depending only on a spherical angle $\theta$. After reviewing the action of supertranslations preserving the static gauge of the metric, we study general transformations preserving the near-horizon form of the metric. We determine the asymptotic Killing vectors and calculate the charge corresponding to the asymptotic symmetries preserving the form of the metric at the horizon.
\end{abstract}

DOI: 10.1103/PhysRevD.99.064052

\section{INTRODUCTION}

The study of asymptotically flat spacetimes initiated by Bondi, Metzner, and Sachs (BMS) [1,2] has attracted much attention recently (for a review, see Ref. [3]). The symmetry group of the asymptotically flat gravity extends the Poincaré group and contains supertranslations, the angular-dependent translations at null infinity, which generalize translations. The vacua with different supertranslation fields are physically different in the sense that their (super-rotation) charges are different [4-9]. Different vacua differ from one another by the cloud of massless particles they contain $[10,12]$.

Of particular interest is the study of black holes containing a supertranslation field. It was suggested that the evaporation of black holes with the inclusion of soft quanta may be unitary [10-12]. Finite supertranslation diffeomorphisms map physical states to inequivalent physical states having different charges. Stationary black holes resulting from the collapse are diffeomorphic to the Kerr metric $[13,14]$ and have the same ADM charges. Because diffeomorphisms contain supertranslations, the metric of a black hole, in general, contains a supertranslation field. The possibility that the account of supertranslation (and superrotation) hair may contribute to solving the information problem has been discussed [15-18].

The BMS transformations are naturally formulated at the null infinity, and there is a complicated problem of

\footnotetext{
iofa@theory.sinp.msu.ru
}

Published by the American Physical Society under the terms of the Creative Commons Attribution 4.0 International license. Further distribution of this work must maintain attribution to the author(s) and the published article's title, journal citation, and DOI. Funded by SCOAP ${ }^{3}$. extension of an asymptotically defined metric with a supertranslation field in a closed form to the bulk. In Ref. [8], a family of vacua containing a supertranslation field was constructed in the bulk. In Ref. [9], a solution-generated technique was developed and applied to the construction of solutions to the Einstein equations containing a supertranslation field generalizing the Schwarzschild metric.

Because many properties of black holes are associated with physics near the horizon, it is natural to study the nearhorizon symmetries of the metric containing the supertranslation field.

We consider the class of metrics containing a supertranslation field depending only on a spherical angle $\theta$. The metrics are solutions of the Einstein equations and are a generalization of the Schwarzschild solution. We transform the metric to a coordinate system in which horizon of the metric is located at $r=2 \mathrm{M}$. A specific property of this metric is that, in contrast to the metrics without a supertranslation field, the expansion of the metric near the horizon is in powers of $(r-2 M)^{1 / 2}$ and not in powers of $r-2 M[19,20]$.

We review the action of supertranslations preserving the gauge of the metric [9]. We find a sufficient condition on supertranslations $T(\theta)$ preserving the gauge of the metric to preserve the near-horizon form of the metric.

We perturbatively solve equations for the asymptotic Killing vectors preserving the functional form of the metric at the horizon (cf. e.g. Refs. [21-28] and many other papers). We calculate the variation of the surface charge corresponding to the asymptotic horizon symmetries. We find that in the case of the black hole with supertranslation field $C(\theta)$ depending only on spherical angle $\theta$ the variation can be integrated to a charge in a closed form. 


\section{SCHWARZSHILD METRIC CONTAINING A SUPERTRANSLATION FIELD}

In this section, we briefly review the exact solution of the Einstein equations generalizing the Schwarzschild metric and containing a supertranslation field constructed in paper [9]. Next, we transform the metric of Ref. [9] to a form with the horizon located at the surface $r=2 M$, where $M$ is interpreted as a mass of black hole.

The solution of Ref. [9] is

$$
\begin{aligned}
d s^{2}= & \tilde{g}_{m n} d x^{m} d x^{n} \\
= & -\frac{\left(1-M / 2 \rho_{s}\right)^{2}}{\left(1+M / 2 \rho_{s}\right)^{2}} d t^{2}+\left(1+M / 2 \rho_{s}\right)^{4}\left(d \rho^{2}+\left(\left((\rho-E)^{2}\right.\right.\right. \\
& \left.\left.+U) \gamma_{A B}+(\rho-E) C_{A B}\right) d z^{A} d z^{B}\right)
\end{aligned}
$$

$C(\theta, \varphi)$ is a supertranslation field, and $C_{00}$ is the lowest spherical harmonic of $C(\theta, \varphi)$. In the following, we do not write $C_{00}$ explicitly understanding $C \rightarrow C-C_{00}$. Here,

$$
\rho_{s}(\rho, C)=\sqrt{(\rho-C)^{2}+D_{A} C D^{A} C} .
$$

Covariant derivatives $D_{A}$ are defined with respect to the metric on the sphere $d s^{2}=d \theta^{2}+\sin ^{2} \theta d \varphi^{2}$. The tensor $C_{A B}$ and the functions $U$ and $E$ are

$$
\begin{aligned}
C_{A B} & =-\left(2 D_{A} D_{B}-\gamma_{A B} D^{2}\right) C, \\
U & =\frac{1}{8} C_{A B} C^{A B}, \\
E & =\frac{1}{2} D^{2} C+C .
\end{aligned}
$$

In the following, we consider the case of metrics with $C$ depending only on angle $\theta$. In this case, the components $C_{A B}$ are

$$
\begin{aligned}
C_{\theta \theta} & =-\left(C^{\prime \prime}-C^{\prime} \cot \theta\right), \\
C_{\varphi \varphi} & =\sin ^{2} \theta\left(C^{\prime \prime}-C^{\prime} \cot \theta\right), \\
C_{\theta \varphi} & =0
\end{aligned}
$$

Here, the prime is differentiation over $\theta$. The horizon of the metric (1) is located at the surface [see Ref. [9], Eq. (45)],

$$
\rho_{H}=C+\sqrt{\frac{M^{2}}{4}-D_{A} C D^{A} C} .
$$

To obtain another form of the metric with horizon located at $r=2 M$, we introduce a new variable, $r=r(\theta, \rho)$, defining

$$
r=\rho_{s}(\rho, C)\left(1+\frac{M}{2 \rho_{s}(\rho, C)}\right)^{2} .
$$

The inverse transformation $\rho=\rho(\theta, r)$ is obtained from the relation

$$
\sqrt{(\rho-C)^{2}+C^{\prime 2}}=\frac{1}{2}(r-M+\sqrt{r(r-2 M)}) .
$$

Introducing the functions

$$
\begin{gathered}
V=1-\frac{2 M}{r}, \\
K=r-M+\sqrt{r(r-2 M)}, \quad \frac{d K}{d r}=\frac{K}{r V^{1 / 2}},
\end{gathered}
$$

we have

$$
\frac{\left(1-M / 2 \rho_{s}\right)^{2}}{\left(1+M / 2 \rho_{s}\right)^{2}}=V, \quad\left(1+M / 2 \rho_{s}\right)^{4}=\frac{4 r^{2}}{K^{2}} \text {. }
$$

Expressing $\rho$ as a function of $r$ and $C$, we obtain

$$
\rho=C+\frac{K}{2}\left(1-\frac{4 C^{\prime 2}}{K^{2}}\right)^{1 / 2} .
$$

Denoting

$$
b=\frac{2 C^{\prime}}{K}
$$

we have

$$
d \rho=\frac{K}{2}\left[\left(b-\frac{b b^{\prime}}{\sqrt{1-b^{2}}}\right) d \theta+\frac{d r}{r V^{1 / 2} \sqrt{1-b^{2}}}\right] .
$$

Because $K(r)$ is an increasing function of $r$ having its minimum at $r=2 M$ and because the cosmic censorship conjecture implies [see Eq. (47) of Ref. [9]) that

$$
1-\frac{C^{\prime 2}}{M^{2}}>0
$$

we have $1-b^{2}>0$. We obtain the line element (1) in the form [29]

$$
\begin{aligned}
d s^{2}= & g_{\mu \nu} d x^{\mu} d x^{\nu} \\
= & -V d t^{2}+\frac{d r^{2}}{V\left(1-b^{2}\right)}+2 d r d \theta \frac{b r\left(\sqrt{1-b^{2}}-b^{\prime}\right)}{\left(1-b^{2}\right) V^{1 / 2}} \\
& +d \theta^{2} r^{2} \frac{\left(\sqrt{1-b^{2}}-b^{\prime}\right)^{2}}{\left(1-b^{2}\right)} \\
& +d \varphi^{2} r^{2} \sin ^{2} \theta\left(b \cot \theta-\sqrt{1-b^{2}}\right)^{2} \\
=- & V d t^{2}+d r^{2} \frac{\bar{g}_{r r}}{V}+2 d r d \theta \frac{\bar{g}_{r \theta}}{V^{1 / 2}}+d \theta^{2} \bar{g}_{\theta \theta}+d \varphi^{2} \sin ^{2} \theta \bar{g}_{\varphi \varphi} .
\end{aligned}
$$

The horizon of the metric (14) is located at the surface $r_{H}=2 M$. In Appendix A, we consider the solution of the 
geodesic equations for null geodesics in the metric (14). We find the asymptotic of the solution in the limit $V(r) \rightarrow 0$. In this limit,

$$
\frac{d r}{d t}=\operatorname{const} V(r)
$$

i.e., the same relation as in the Schwarzschild metric. The surface $r=2 M$ is the surface of the infinite redshift, i.e., the horizon [30]. In the following, we set $M=1$.

\section{SUPERTRANSLATIONS PRESERVING THE GAUGE OF THE METRIC}

In this section, first, we review the supertranslations preserving the gauge of the metric (1). Next, we write the generator of supertranslations preserving the gauge of the metric (14). Imposing the requirement that supertranslations do not alter the functional form of the metric at the horizon, we determine necessary conditions on supertranslations to preserve the near-horizon form of the metric. We begin with the metric component $g_{t t}$ and find conditions on supertranslations under which the functional form of $g_{t t}$ at the horizon is preserved. Considering other components of the metric, we show that the condition obtained for $g_{t t}$ is sufficient to preserve the near-horizon form of all components.

The metric (1) is written in the static gauge $\tilde{g}_{t \rho}=\tilde{g}_{t \theta}=0$ and $\tilde{g}_{\rho a}=0$. The generator of supertranslations preserving this gauge was obtained in Ref. [9] and in the case $C=$ $C(\theta)$ is

$$
\xi_{T}=T_{00} \frac{\partial}{\partial t}-\left(T-T_{00}\right) \frac{\partial}{\partial \rho}-\frac{T^{\prime}}{\rho-C-C^{\prime \prime}} \frac{\partial}{\partial \theta} .
$$

In the following, we include $T_{00}$ in $T$, writing simply $T$. The metric components transform as

$L_{\xi_{T}} \tilde{g}_{\mu \nu}=\lim _{\varepsilon=0}\left[\tilde{g}_{\mu \nu}(C+\varepsilon T)-\tilde{g}_{\mu \nu}(C)\right] / \varepsilon \equiv \delta_{T} \tilde{g}_{\mu \nu}(C)$,

where $\delta_{T} C=T$ and $\delta_{T} D^{k} C=D^{k} T$. The action of the transformation (16) on the component $\tilde{g}_{t t}$ is

$$
L_{\xi_{T}} \tilde{g}_{t t}=4 \frac{\rho_{s}-1 / 2}{\left(\rho_{s}+1 / 2\right)^{3}} \delta_{T} \rho_{s}
$$

where

$$
\delta_{T} \rho_{s}=\frac{-T(\rho-C)+C^{\prime} T^{\prime}}{\rho_{s}} .
$$

The horizon of the metric (1) is located at the surface $\rho_{s}=1 / 2$.

Transformations (16) form a commutative algebra with respect to the modified bracket $[4,5,9]$

$$
\left[\xi_{1}, \xi_{2}\right]_{\bmod }=\left[\xi_{1}, \xi_{2}\right]-\delta_{T_{1}} \xi_{2}+\delta_{T_{2}} \xi_{1}
$$

Because $\left[\xi_{1}, \xi_{2}\right]^{\rho}=0$ and $\delta_{T_{1}} \xi_{2}^{\rho}=0$, the commutator $\left[\xi_{1}, \xi_{2}\right]_{\bmod }^{\rho}$ is zero. The commutator $\left[\xi_{1}, \xi_{2}\right]^{\theta}$ is equal to

$$
\left[\xi_{1}, \xi_{2}\right]^{\theta}=\frac{T_{12}+T_{12}^{\prime}}{\left(\rho-C-C^{\prime \prime}\right)^{2}},
$$

where $T_{12}=T_{2} T_{1}^{\prime}-T_{1} T_{2}^{\prime}$. Because

$$
\delta_{T_{1}} \xi_{2}^{\theta}=-T_{2}^{\prime} \frac{T_{1}+T_{1}^{\prime \prime}}{\left(\rho-C-C^{\prime \prime}\right)^{2}},
$$

we obtain for $\left[\xi_{1}, \xi_{2}\right]_{m o d}^{\theta}$ zero result.

Next, we consider supertranslations acting on the metric (14). The generator of supertranslations in variables $(r, \theta)$ acting on the metric (14) is obtained from the generator (16) by transformation

$$
\begin{aligned}
& \chi_{T}^{r}=\xi^{\rho} \frac{\partial r}{\partial \rho}+\xi^{\theta} \frac{\partial r}{\partial \theta}=\xi^{\rho} \frac{\partial r}{\partial \rho_{s}} \frac{\partial \rho_{s}}{\partial \rho}+\xi^{\theta} \frac{\partial r}{\partial \rho_{s}} \frac{\partial \rho_{s}}{\partial \theta} \\
& \chi_{T}^{\theta}=\xi^{\rho} \frac{\partial \theta}{\partial \rho}+\xi^{\theta} \frac{\partial \theta}{\partial \theta}=\xi_{T}^{\theta} .
\end{aligned}
$$

Using (6) to calculate $\partial r / \partial \rho_{s}=1-1 /\left(4 \rho_{s}^{2}\right)$ and expressing $\chi_{T}^{i}$ through $r, \theta$, we obtain

$$
\begin{aligned}
\chi_{T}= & T_{00} \frac{\partial}{\partial t}+\frac{K^{2}-1}{K^{2}}\left(-T \sqrt{1-b^{2}}+T^{\prime} b\right) \frac{\partial}{\partial r} \\
& -\frac{2 T^{\prime}}{K\left(\sqrt{1-b^{2}}-b^{\prime}\right)} \frac{\partial}{\partial \theta} .
\end{aligned}
$$

In the near-horizon region, denoting $r=2+x,|x| \ll 1$, we have

$$
\begin{aligned}
K & \simeq 1+\sqrt{2 x}, \quad b=b_{0}(1-\sqrt{2 x}), \\
b_{0} & =2 C^{\prime}, \quad \partial K / \partial r \simeq 1 / \sqrt{2 x}
\end{aligned}
$$

The action of the generator of supertranslations on the component $g_{t t}$ of the metric (14) yields

$$
L_{\chi_{T}} g_{t t}=\frac{2}{r^{2}} \frac{K^{2}-1}{K^{2}}\left(-T \sqrt{1-b^{2}}+T^{\prime} b\right) .
$$

In the near-horizon region,

$$
g_{t t}=\frac{x}{2}+O\left(x^{2}\right)
$$

To preserve the functional form (26) of the metric component at $x \rightarrow 0$, the transformed component (25) should be of order $O(x)$, or less. Because $\left(K^{2}-1\right) / K^{2}=O\left(x^{1 / 2}\right)$, the sufficient condition is 


$$
-T \sqrt{1-b^{2}}+T^{\prime} b=O\left(x^{1 / 2}\right)
$$

and

$$
\frac{K^{2}-1}{K^{2}}\left(-T \sqrt{1-b^{2}}+T^{\prime} b\right)=O(x) .
$$

The action of the term $\chi_{T}^{\theta} \partial_{\theta}$ on the metric components does not change their behavior as $x \rightarrow 0$. Let us consider the action of $\chi_{T}^{r} \partial_{r}$. When acting on $\bar{g}_{r r}, \bar{g}_{r \theta}, \bar{g}_{\theta \theta}, \bar{g}_{\varphi \varphi}$, the operator $\chi_{T}^{r} \partial_{r}$ produces the terms proportional to $V^{-1 / 2}$. When acting on the terms $V^{-1}$ and $V^{-1 / 2}$, the operator $\chi_{T}^{r}$ produces an extra factor $V^{-1}$. However, because of condition (28), the total singularity of each term remains the same or is reduced.

Condition (27) gives a restriction on supertranslation $T(\theta)$ :

$$
-T \sqrt{1-b_{0}^{2}}+T^{\prime} b_{0}=0
$$

Using (29), we obtain

$$
-T \sqrt{1-b^{2}}+T^{\prime} b=-\frac{\sqrt{2 x} T}{\sqrt{1-b_{0}^{2}}}=O\left(x^{1 / 2}\right) .
$$

To obtain the relation (30), we have used (29). Equation (29) is the first-order equation on $T(\theta) . T(\theta)$ is expressed through $C(\theta)$ and is determined up to an arbitrary constant.

\section{ASYMPTOTIC HORIZON SYMMETRIES OF THE METRIC}

In this section, we consider general diffeomorphisms which do not change the form of the metric components in the near-horizon region. We find transformations of the leading-order parts of the metric components.

At $x=r-2 \ll 1$, the metric (14) takes the form

$$
\begin{aligned}
d s^{2}= & \left(-\kappa x+O\left(x^{2}\right)\right) d t^{2}+\left(\frac{g_{r r,-1}}{x}+O\left(x^{-1 / 2}\right)\right) d x^{2} \\
& +2\left(\frac{g_{r \theta,-1 / 2}}{x^{1 / 2}}+O\left(x^{0}\right)\right) d x d \theta+\left(g_{\theta \theta, 0}+O\left(x^{1 / 2}\right) d \theta^{2}\right. \\
& +\left(g_{\varphi \varphi, 0}+O\left(x^{1 / 2}\right)\right) d \varphi^{2}
\end{aligned}
$$

The coefficients of expansions of the metric components in series in $x^{1 / 2}$ are functions of $\theta$. Here, $\kappa$ is introduced to treat the component $g_{t t}$ on the same footing as other components. In the final expressions, $\kappa$ is set to unity.

We consider transformations generated by vector fields $\chi^{i}$ which do not change the form of the metric components in the leading orders. The vector fields generating the nearhorizon transformations have the following structure:

$$
\chi^{k}=\chi_{0}^{k}+x^{1 / 2} \chi_{1 / 2}^{k}+x \chi_{1}^{k}+\cdots
$$

Under the diffeomorphisms generated by vector fields $\chi^{k}$, the metric components are transformed as

$$
L_{\chi} g_{m n}=\chi^{k} \partial_{k} g_{m n}+\partial_{m} \chi^{k} g_{k n}+\partial_{n} \chi^{k} g_{k m}
$$

Transformations preserving the gauge conditions $g_{r t}=$ $g_{\theta t}=0$ are

$$
\begin{aligned}
& L_{\chi} g_{r t}=\partial_{r} \chi^{t} g_{t t}+\partial_{t} \chi^{r} g_{r r}+\partial_{t} \chi^{\theta} g_{\theta r}=0, \\
& L_{\chi} g_{\theta t}=\partial_{\theta} \chi^{t} g_{t t}+\partial_{t} \chi^{r} g_{r \theta}+\partial_{t} \chi^{\theta} g_{\theta \theta}=0 .
\end{aligned}
$$

Other components of the metric transform as

$$
L_{\chi} g_{t t}=\chi^{r} \partial_{r} g_{t t}+\chi^{\theta} \partial_{\theta} g_{t t}+2 \partial_{t} \chi^{t} g_{t t}=O(x),
$$

$$
\begin{aligned}
L_{\chi} g_{r r} & =\chi^{r} \partial_{r} g_{r r}+\chi^{\theta} \partial_{\theta} g_{r r}+2 \partial_{r} \chi^{r} g_{r r}+\partial_{r} \chi^{\theta} g_{r \theta} \\
& =O(1 / x)
\end{aligned}
$$

$$
L_{\chi} g_{r \theta}=\chi^{r} \partial_{r} g_{r \theta}+\chi^{\theta} \partial_{\theta} g_{r \theta}+\partial_{\theta} \chi^{r} g_{r r}+\partial_{\theta} \chi^{\theta} g_{r \theta}
$$$$
+\partial_{r} \chi^{r} g_{r \theta}+\partial_{r} \chi^{\theta} g_{\theta \theta}=O\left(x^{-1 / 2}\right) \text {, }
$$

$L_{\chi} g_{\theta \theta}=\left(\chi^{r} \partial_{r}+\chi^{\theta} \partial_{\theta}\right) g_{\theta \theta}+2 \partial_{\theta} \chi^{r} g_{r \theta}+2 \partial_{\theta} \chi^{\theta} g_{\theta \theta}=O\left(x^{0}\right)$.

From Eq. (37), it follows that

$$
\chi_{0}^{r}=\chi_{1 / 2}^{r}=0
$$

From Eqs. (34) and (35), we obtain

$$
\dot{\chi}_{0}^{\theta}=\dot{\chi}_{1 / 2}^{\theta}=\dot{\chi}_{1}^{r}=0 \text {. }
$$

Here, the dot is differentiation over $t$.

Transformations of the leading-order parts of the metric components are

$$
\begin{aligned}
\delta \kappa= & \chi_{1}^{r} \kappa+\chi_{0}^{\theta} \partial_{\theta} \kappa+2 \dot{\chi}_{0}^{t} \kappa \\
\delta g_{r r,-1}= & \chi_{1}^{r} g_{r r,-1}+\chi_{1 / 2}^{\theta} g_{\theta r,-1 / 2}+\chi_{0}^{\theta} \partial_{\theta} g_{r r,-1}, \\
\delta g_{\theta r,-1 / 2}= & \frac{1}{2} \chi_{1}^{r} g_{\theta r,-1 / 2}+\chi_{0}^{\theta} g_{\theta r,-1 / 2}+\partial_{\theta} \chi_{0}^{\theta} g_{\theta r,-1 / 2} \\
& +\frac{1}{2} \chi_{1 / 2}^{\theta} g_{\theta \theta, 0}, \\
\delta g_{\theta \theta, 1 / 2}= & \frac{1}{2} \chi_{1}^{r} g_{\theta \theta, 1 / 2}+2 \partial_{\theta} \chi_{1}^{r} g_{r \theta,-1 / 2}+\chi_{0}^{\theta} \partial_{\theta} g_{\theta \theta, 1 / 2} \\
& +\chi_{1 / 2}^{\theta} \partial_{\theta} g_{\theta \theta, 0}+2 \partial_{\theta} \chi_{0}^{\theta} g_{\theta \theta, 1 / 2}+2 \partial_{\theta} \chi_{1 / 2}^{\theta} g_{\theta \theta, 0} \\
\delta g_{\varphi \varphi, 0}= & \chi^{\theta} \partial_{\theta} g_{\varphi \varphi, 0} .
\end{aligned}
$$

It is seen that the leading-order parts of the metric components are transformed through the functions 


$$
\chi_{1}^{r}(\theta), \quad \chi_{0}^{\theta}(\theta), \quad \chi_{1 / 2}^{\theta}(\theta)
$$

and $\dot{\chi}_{0}^{t}$.

The vector fields in the generator of supertranslations (23) satisfying condition (27) form a subset of the vector fields (32)

$$
\begin{gathered}
\chi^{r}=x \chi_{1}^{r}=-x \frac{T}{\sqrt{1-b_{0}^{2}}}, \\
\chi^{\theta}=\chi_{0}^{\theta}=-\frac{T^{\prime}}{\sqrt{1-b_{0}^{2}}-b_{0}^{\prime}} .
\end{gathered}
$$

The Lie brackets of the vector fields in which we retain the components (43) are

$$
\begin{aligned}
{\left[\chi_{(1)}, \chi_{(2)}\right]_{0}^{t}=} & \chi_{(12), 0}^{t}=\chi_{(1), 0}^{t} \stackrel{\leftrightarrow}{\partial}_{t} \chi_{(2), 0}^{t}+\chi_{(1), 0}^{\theta} \partial_{\theta} \chi_{(2), 0}^{t} \\
& -\chi_{(2), 0}^{\theta} \partial_{\theta} \chi_{(1), 0}^{t} \\
{\left[\chi_{(1)}, \chi_{(2)}\right]_{1}^{r}=} & \chi_{(12), 1}^{r}=\chi_{(1), 0}^{\theta} \partial_{\theta} \chi_{(2), 1}^{r}-\chi_{(2), 0}^{\theta} \partial_{\theta} \chi_{(1), 1}^{r} \\
{\left[\chi_{(1)}, \chi_{(2)}\right]_{0}^{\theta}=} & \chi_{(12), 0}^{\theta}=\chi_{(1), 0}^{\theta} \stackrel{\leftrightarrow}{\partial}_{\theta} \chi_{(2), 0}^{\theta} \\
{\left[\chi_{(1)}, \chi_{(2)}\right]_{1 / 2}^{\theta}=} & \chi_{(1), 0}^{\theta} \partial_{\theta} \chi_{(2), 1 / 2}^{\theta}-\chi_{(2), 0}^{\theta} \partial_{\theta} \chi_{(1), 1 / 2}^{\theta} \\
& +1 / 2\left(\chi_{(1), 1}^{r} \chi_{(2), 1 / 2}^{\theta}-\chi_{(2), 1}^{r} \chi_{(1), 1 / 2}^{\theta}\right) .
\end{aligned}
$$

In the next section, we use transformations (42) to calculate the surface charge of the asymptotic horizon symmetries.

\section{SURFACE CHARGE OF ASYMPTOTIC HORIZON SYMMETRIES}

In this section, we calculate the variation of the surface charge corresponding to the asymptotic Killing vector field $\chi^{i}$. The metric variations needed to obtain the variation of the charge were obtained in (42). Details of the calculation are presented in Appendix B. In our calculations, we used the relations specific to the case of $C$ depending only on $\theta$.

Let us introduce compact notations for the metric components in (31):

$$
g_{\mu \nu}=\left|\begin{array}{cccc}
-\kappa x & 0 & 0 & 0 \\
0 & \bar{g}_{r r} / x & \bar{g}_{r \theta} / \sqrt{x} & 0 \\
0 & \bar{g}_{r \theta} / \sqrt{x} & \bar{g}_{\theta \theta} & 0 \\
0 & 0 & 0 & \bar{g}_{\varphi \varphi} \sin ^{2} \theta
\end{array}\right| .
$$

The determinant of the matrix (50) is

$$
|\operatorname{detg}|=\kappa\left(\bar{g}_{r r} \bar{g}_{\theta \theta}-\bar{g}_{r \theta}^{2}\right) \bar{g}_{\varphi \varphi} \sin ^{2} \theta=\kappa \bar{g}_{\varphi \varphi} \bar{g}_{\theta \theta} \sin ^{2} \theta .
$$

To obtain (51), we have used the relation

$$
\bar{g}_{r r} \bar{g}_{\theta \theta}-\bar{g}_{r \theta}^{2}=\bar{g}_{\theta \theta}
$$

The inverse metric is

$$
g^{\mu \nu}=\left|\begin{array}{cccc}
(-x \kappa)^{-1} & 0 & 0 & 0 \\
0 & x & -\sqrt{x} \bar{g}_{r \theta} / \bar{g}_{\theta \theta} & 0 \\
0 & -\sqrt{x} \bar{g}_{r \theta} / \bar{g}_{\theta \theta} & \bar{g}_{r r} / \bar{g}_{\theta \theta} & 0 \\
0 & 0 & 0 & \left(\bar{g}_{\varphi \varphi} \sin ^{2} \theta\right)^{-1}
\end{array}\right| .
$$

The matrix of metric variations is

$$
h_{\mu \nu} \equiv \delta g_{\mu \nu}=\left|\begin{array}{cccc}
-\delta \kappa x & 0 & 0 & 0 \\
0 & \bar{h}_{r r} / x & \bar{h}_{r \theta} / x^{1 / 2} & 0 \\
0 & \bar{h}_{r \theta} / x^{1 / 2} & \bar{h}_{\theta \theta} & 0 \\
0 & 0 & 0 & \bar{h}_{\varphi \varphi} \sin ^{2} \theta
\end{array}\right| .
$$

Variations with the upper indices are defined as $h^{\mu \nu}=$ $g^{\mu \rho} \delta g_{\rho \lambda} g^{\lambda \nu}$. Using relation (52) again, we find that $h^{r r}=0$ :

$$
\begin{aligned}
h^{r r} & =\left(g^{r r}\right)^{2} \delta g_{r r}+2 g^{r r} g^{r \theta} \delta g_{r \theta}+\left(g^{r \theta}\right)^{2} \delta g_{\theta \theta} \\
& =x^{2} \delta\left(\bar{g}_{r r}-\frac{\bar{g}_{r \theta}^{2}}{\bar{g}_{\theta \theta}}\right)=0 .
\end{aligned}
$$

Taking the trace of variations, we have

$$
\begin{aligned}
h=h_{\mu \nu} g^{\mu \nu} & =\frac{\delta \kappa}{\kappa}+\delta \bar{g}_{r r} \bar{g}^{r r}+2 \delta \bar{g}_{r \theta} \bar{g}^{r \theta}+\delta \bar{g}_{\theta \theta} \bar{g}^{\theta \theta}+\delta \bar{g}_{\varphi \varphi} \bar{g}^{\varphi \varphi} \\
& =\frac{\delta \kappa}{\kappa}+\frac{\delta\left(\bar{g}_{r r} \bar{g}_{\theta \theta}-\bar{g}_{r \theta}^{2}\right)}{\bar{g}_{\theta \theta}}+\frac{\delta \bar{g}_{\varphi \varphi}}{\bar{g}_{\varphi \varphi}}=\frac{\delta \kappa}{\kappa}+\frac{\delta \bar{g}_{\theta \theta}}{\bar{g}_{\theta \theta}}+\frac{\delta \bar{g}_{\varphi \varphi}}{\bar{g}_{\varphi \varphi}} .
\end{aligned}
$$

The variation of the surface charge is $[31,32]$

$$
\not Q_{\chi}(g, h)=\frac{1}{16 \pi} \int\left(d^{2} x\right)_{\mu \nu} F^{\mu \nu},
$$

where $\left(d^{2} x\right)_{\mu \nu}=1 / 4 \varepsilon_{\mu \nu \alpha \beta} d x^{\alpha} \wedge d x^{\beta}$. Explicitly,

$$
\begin{aligned}
\not \hat{Q}_{\chi}(g, h) & =\frac{1}{4 \pi} \int\left(d^{2} x\right)_{r t} \sqrt{g^{(2)}}\left[\chi^{r} \nabla^{t} h-\chi^{r} \nabla_{\sigma} h^{t \sigma}+\chi_{\sigma} \nabla^{r} h^{t \sigma}\right. \\
& \left.+\frac{1}{2} h \nabla^{r} \chi^{t}+\frac{1}{2} h^{r \sigma}\left(\nabla^{t} \chi_{\sigma}-\nabla_{\sigma} \chi^{t}\right)-(r \leftrightarrow t)\right] .
\end{aligned}
$$

Calculating the integrand $F^{r t}$, we obtain 


$$
\begin{aligned}
F^{r t}= & \frac{\chi_{0}^{t}}{2}\left[0 /-\frac{\delta \kappa}{\kappa} /+\frac{\delta \kappa}{\kappa} /+\frac{\delta \kappa}{\kappa}+\frac{\delta \bar{g}_{\theta \theta}}{\bar{g}_{\theta \theta}}+\frac{\delta \bar{g}_{\varphi \varphi}}{\bar{g}_{\varphi \varphi}} /-\frac{\delta \kappa}{2 \kappa} /-\frac{\delta \kappa}{2 \kappa}\right] \\
& +O\left(x^{1 / 2}\right) \\
= & \left.\frac{\chi_{0}^{t}}{2}\left(\frac{\delta \bar{g}_{\theta \theta}}{\bar{g}_{\theta \theta}}+\frac{\delta \bar{g}_{\varphi \varphi}}{\bar{g}_{\varphi \varphi}}\right)\right|_{x=0}+O\left(x^{1 / 2}\right) .
\end{aligned}
$$

Here, / divides contributions $O\left(x^{0}\right)$ from the consecutive terms in (58). Details of the calculation are presented in Appendix B.

Substituting the above expressions, we obtain the variation of the surface charge,

$$
\begin{aligned}
\not \hat{Q} & =\frac{1}{16 \pi} \int d \varphi d \theta \sin \theta \chi_{0}^{t}\left(\kappa \bar{g}_{\theta \theta} \bar{g}_{\varphi \varphi}\right)^{1 / 2}\left(\frac{\delta \bar{g}_{\theta \theta}}{\bar{g}_{\theta \theta}}+\frac{\delta \bar{g}_{\varphi \varphi}}{\bar{g}_{\varphi \varphi}}\right) \\
& =\frac{1}{16 \pi} \int d \varphi d \theta \sin \theta \chi_{0}^{t} \kappa^{1 / 2} \frac{\delta\left(\bar{g}_{\theta \theta} \bar{g}_{\varphi \varphi}\right)}{\left(\bar{g}_{\theta \theta} \bar{g}_{\varphi \varphi}\right)^{1 / 2}} .
\end{aligned}
$$

Here, integration is over the surface $r=2$. Setting $\kappa=1$ in correspondence with the metric (14) and integrating the variation (60), we obtain

$$
\hat{Q}=\frac{1}{8 \pi} \int d \varphi d \theta \sin \theta \chi_{0}^{t}\left(\bar{g}_{\theta \theta} \bar{g}_{\varphi \varphi}\right)^{1 / 2}+\hat{Q}_{0} .
$$

If $\chi_{0}^{t}$ is independent of $\theta$, the surface charge is proportional to the surface of the horizon, i.e., to the geometric entropy of the black hole.

The Lie bracket of the charges is

$$
\left[\hat{Q}\left(\chi_{(1), 0}^{t}\right), \hat{Q}\left(\chi_{(2), 0}^{t}\right)\right]=\hat{Q}\left(\chi_{(12), 0}^{t}\right),
$$

where $\chi_{(12), 0}^{t}$ is defined in (46).

\section{CONCLUSIONS}

In this paper, we studied the horizon symmetries of the metric containing a supertranslation field depending only on spherical angle $\theta$. We transformed the metric obtained in Ref. [9] to the form with a horizon located at the surface $r=2 M$. After reviewing the action of supertranslations preserving the form of the metric [9], we determined the requirements on the generator of supertranslations to preserve the near-horizon form of the metric. It was found that to preserve the functional form of the metric in the near-horizon region, at $r-2 M \ll 1$, the $r$ component of the generator of supertranslations should be of order $O(r-2 M)$. From this requirement, it follows that the parameter of supertranslations, $T(\theta)$, must satisfy a condition,

$$
-T(\theta) \sqrt{1-4 C^{\prime}(\theta)^{2}}+2 T^{\prime}(\theta) C^{\prime}(\theta)=0 .
$$

Next, we studied the general transformations preserving the near-horizon form of the metric and calculated the charge corresponding to the asymptotic horizon Killing symmetries. In calculating the variation of the charge, we used the relations between the metric components specific to the case of the supertranslation field depending only on angle $\theta$. In the case of the supertranslation field depending only on $\theta$, the variation of the charge can be integrated to a charge in a closed form. The charge is proportional to the area of horizon surface, i.e., to the geometric entropy of a black hole.

In the literature (a partial list is Refs. [21-28]) and in many subsequent papers, the near-horizon symmetries have been studied for the metrics with the isolated horizon, which near the horizon have the form

$$
\begin{aligned}
d s^{2}= & -2 \kappa \rho d v^{2}+2 d v d \rho+2 \theta_{a} \rho d v d x^{a} \\
& +\left(\Omega \gamma_{a b}+\lambda_{a b} \rho\right) d x^{a} d x^{b} .
\end{aligned}
$$

The metric is written in the gauge $g_{\rho \rho}=g_{v \rho}=g_{\rho a}=0$ with accuracy $O\left(\rho^{2}\right)$. The horizon of the metric is located at $\rho=0$. In the near-horizon region, the metric components are expanded in power series in $\rho$. The charge of the asymptotic near-horizon symmetries for the metric (63) was obtained in Refs. [25,26] in the form

$$
Q=\frac{1}{16 \pi G} \int d z d \bar{z} \sqrt{|\gamma|}\left(2 T \kappa \Omega-y^{a} \theta_{a} \Omega\right) .
$$

Here, $T$ is a part of the asymptotic Killing vector $\chi^{v}$. The volume $\sqrt{\gamma} \Omega d z d \bar{z}$, where $\Omega_{z \bar{z}} \sim \Omega \gamma_{z \bar{z}}$, is an analog of the volume $\sin \theta\left(\bar{g}_{\theta \theta} \bar{g}_{\varphi \varphi}\right)^{1 / 2} d \theta d \varphi$ in (61), and $T$ is an analog of $\chi^{t}$. It is seen that structures of charges in both cases are similar; however, we could not transform the metrics of the form (14) considered in the present paper to the form (63).

In contrast to the metric (63), the components of the metric (14) are expanded in powers of $x^{1 / 2}=(r-2 M)^{1 / 2}$. In the limit of zero supertranslation field, the terms with fractional powers of $(r-2 M)$ vanish.

\section{ACKNOWLEDGMENTS}

I thank Misha Smoliakov and Igor Volobuev for interesting discussion. This work was partially supported by the Ministry of Science and Higher Education of Russian Federation under the project 01201255504.

\section{APPENDIX A}

In this Appendix, we consider a solution of the geodesic equations in the metric (14). We find an asymptotic of a solution for null geodesic in the limit $V(r) \rightarrow 0$. Following the treatment of Ref. [33], we start from the Lagrangian corresponding to the metric (15)

$$
2 \mathcal{L}=-V \dot{t}^{2}+\frac{\dot{r}^{2}}{V} \bar{g}_{r r}+2 \frac{\dot{r} \dot{\theta}}{V^{1 / 2}} \bar{g}_{r \theta}+\dot{\theta}^{2} \bar{g}_{\theta \theta}+\dot{\varphi}^{2} \sin ^{2} \theta \bar{g}_{\varphi \varphi} .
$$


The derivatives are taken with respect to an affine parameter on geodesic $\tau$. The function $\varphi$ is the cyclic variable, which can be set equal to zero [33]. The Lagrange equations for null geodesics are

$$
\begin{gathered}
\frac{d(V \dot{t})}{d \tau}=0, \\
\frac{\ddot{r} \bar{g}_{r r}}{V}-\frac{\dot{r}^{2} \bar{g}_{r r, r} V_{, r}}{2 V^{2}}+\frac{\dot{r}^{2} \bar{g}_{r r, r}}{2 V}+\frac{\dot{r} \dot{\theta} \bar{g}_{r r, \theta}}{V}+\frac{\ddot{\theta} \bar{g}_{r \theta}}{V^{1 / 2}} \\
+\frac{\dot{\theta}^{2} \bar{g}_{r \theta, \theta}}{V^{1 / 2}}-\frac{\dot{\theta}^{2} \bar{g}_{\theta \theta, r}}{2}+\frac{\dot{t}^{2} V_{, r}}{2}=0, \\
\frac{\ddot{r} \bar{g}_{r \theta}}{V^{1 / 2}}-\frac{\dot{r}^{2} \bar{g}_{r \theta} V_{, r}}{2 V^{3 / 2}}+\frac{\dot{r}^{2} \bar{g}_{r \theta, r}}{V^{1 / 2}}-\frac{\dot{r}^{2} \bar{g}_{r \theta, r}}{V}+\ddot{\theta} \bar{g}_{\theta \theta}+\dot{r} \dot{\theta} \bar{g}_{\theta \theta, r} \\
+\frac{\dot{\theta}^{2} \bar{g}_{\theta \theta, \theta}}{2}=0 .
\end{gathered}
$$

The system of equations admits the first integral

$$
-\frac{E^{2}}{2 V}+\frac{\dot{r}^{2} \bar{g}_{r r}}{2 V}+\frac{\dot{r} \dot{\theta} \bar{g}_{r \theta}}{V^{1 / 2}}+\frac{\dot{\theta}^{2} \bar{g}_{\theta \theta}}{2}=0 .
$$

We look for the solution in the limit $V(r) \rightarrow 0$ in the form

$$
\begin{gathered}
\dot{t}=\frac{E}{V} \\
\dot{r}=C+C_{1} V^{1 / 2}+\cdots \\
\dot{\theta}=\frac{A}{V^{1 / 2}}+A_{1}+\cdots
\end{gathered}
$$

In the limit $V=0$, we have $r=2 M=2, V_{, r}=2 M / r^{2}=$ $1 / 2$. Substituting the ansatz in the Lagrange equations and retaining the leading in the limit $V \rightarrow 0$ terms, we have

$$
\begin{gathered}
-\frac{C^{2} \bar{g}_{r r}}{4 V^{2}}+\frac{E^{2}}{4 V^{2}}-\frac{A C \bar{g}_{r \theta}}{4 V^{2}}=0, \\
-\frac{C^{2} \bar{g}_{r \theta}}{4 V^{3 / 2}}-\frac{A C \bar{g}_{\theta \theta}}{4 V^{3 / 2}}=0 .
\end{gathered}
$$

From the system (A9) and (A10), it follows that

$$
\begin{gathered}
C \bar{g}_{r \theta}+2 A \bar{g}_{\theta \theta}=0, \\
E^{2}-C^{2}\left(\bar{g}_{r r}-\frac{\bar{g}_{r \theta}^{2}}{\bar{g}_{\theta \theta}}\right)=0 .
\end{gathered}
$$

Substituting the ansatz in relation (A5), we reduce it to

$$
V^{-1}\left[C^{2} \bar{g}_{r r}-E^{2}+2 r C A \bar{g}_{r \theta}+r^{2} A^{2} \bar{g}_{\theta \theta}\right]=0 .
$$

Using the relation (52),

$$
\bar{g}_{r r} \bar{g}_{\theta \theta}-\bar{g}_{r \theta}^{2}=\bar{g}_{\theta \theta}
$$

from Eq. (A12), we find that

$$
C^{2}=E^{2}
$$

In the ansatz (A7), (A8) the relation (A13) is satisfied identically. From (A6) and (A8) in the main order in $V \rightarrow 0$, we obtain

$$
\frac{d r}{d t}= \pm|E| V(r)
$$

From this relation, it follows that the surface $r=2 M$ is the surface of infinite redshift [30].

\section{APPENDIX B}

In this Appendix, we present some details of the calculation of the variation of the surface charge. The integrand in $(60)$ is

$$
\begin{aligned}
F^{r t}= & {\left[\chi^{r} \nabla^{t} h-\chi^{r} \nabla_{\sigma} h^{t \sigma}+\chi_{\sigma} \nabla^{r} h^{t \sigma}+\frac{1}{2} h \nabla^{r} \chi^{t}\right.} \\
& \left.+\frac{1}{2} h^{r \sigma}\left(\nabla^{t} \chi_{\sigma}-\nabla_{\sigma} \chi^{t}\right)-(r \leftrightarrow t)\right] .
\end{aligned}
$$

The first term is

$$
\chi^{r} \nabla^{t} h-\chi^{t} \nabla^{r} h=-\chi^{t}\left(x \partial_{x}-\frac{x^{1 / 2} \bar{g}_{r \theta}}{\bar{g}_{\theta \theta}} \partial_{\theta}\right) h=O\left(x^{1 / 2}\right) .
$$

In the limit $x=0$, its contribution is zero. The second term is

$$
\begin{aligned}
-\chi^{r} \nabla_{\sigma} h^{t \sigma}+\chi^{t} \nabla_{\sigma} h^{r \sigma} & =\chi^{t} \nabla_{\sigma} h^{r \sigma}+O\left(x^{1 / 2}\right) \\
& =-\frac{\chi^{t}}{2} \frac{\delta \kappa}{\kappa}+O\left(x^{1 / 2}\right) .
\end{aligned}
$$

The third term reduces to

$$
\begin{aligned}
\chi_{\sigma} \nabla^{r} h^{t \sigma}-\chi_{\sigma} \nabla^{t} h^{r \sigma} & =\chi_{t}\left(g^{r \theta} \nabla_{\theta} h^{t t}-g^{t t} \nabla_{t} h^{r t}\right)+O\left(x^{1 / 2}\right) \\
& =-\chi^{t} \nabla_{t} h^{r t}+O\left(x^{1 / 2}\right) \\
& =\frac{\chi^{t}}{2} \frac{\delta \kappa}{\kappa}+O\left(x^{1 / 2}\right)
\end{aligned}
$$

The fourth term is

$$
\begin{aligned}
\frac{h}{2}\left(\nabla^{r} \chi^{t}-\nabla^{t} \chi^{r}\right) & =\frac{h}{2}\left(\left(g^{r r} \nabla_{r}-g^{r \theta} \nabla_{\theta}\right) \chi^{t}-g^{t t} \nabla_{t} \chi^{r}\right) \\
& =\frac{\chi^{t}}{2}\left(\frac{\delta \kappa}{\kappa}+\frac{\delta \bar{g}_{\theta \theta}}{\bar{g}_{\theta \theta}}+\frac{\delta \bar{g}_{\varphi \varphi}}{\bar{g}_{\varphi \varphi}}\right)+O\left(x^{1 / 2}\right) .
\end{aligned}
$$

The fifth term yields 


$$
\begin{aligned}
\frac{1}{2}\left(h^{r \sigma} \nabla^{t} \chi_{\sigma}-h^{t \sigma} \nabla^{r} \chi_{\sigma}\right) & =\frac{1}{2}\left(h^{r r} \nabla^{t} \chi_{r}+h^{r \theta} \nabla^{t} \chi_{\theta}-h^{t t} \nabla^{r} \chi_{t}\right) \\
& =\left[\nabla_{t} \chi^{r} g^{t t}\left(h^{r r} g_{r r}+h^{r \theta} g_{r \theta}\right)+\nabla_{t} \chi^{\theta} g^{t t}\left(h^{r r} g_{r \theta}+h^{r \theta} g_{\theta \theta}\right)-h^{t t} g_{t t}\left(g^{r r} \nabla_{r} \chi^{t}+g^{r \theta} \nabla_{\theta} \chi^{t}\right)\right]+O\left(x^{1 / 2}\right) \\
& =-\frac{\chi^{t}}{2} \frac{\delta \kappa}{2 \kappa}+O\left(x^{1 / 2}\right) .
\end{aligned}
$$

The sixth term is

$$
-\frac{1}{2}\left(h^{r \sigma} \nabla_{\sigma} \chi^{t}+h^{t \sigma} \nabla_{r} \chi^{\sigma}\right)=\frac{1}{2}\left[h^{t t} \nabla_{t} \chi^{r}-h^{r r} \nabla_{r} \chi^{t}-h^{r \theta} \nabla_{\theta} \chi^{t}\right]+O\left(x^{1 / 2}\right)=-\frac{\chi^{t}}{2} \frac{\delta \kappa}{2 \kappa}+O\left(x^{1 / 2}\right) .
$$

[1] H. Bondi, M. G. J. van der Burg, and A. W. K. Metzner, Gravitational waves in general relativity 7, Proc. R. Soc. A 269, 21 (1962).

[2] R. K. Sachs, Gravitational waves in general relativity 8 . Waves in asymptotically flat space-time, Proc. R. Soc. Edinburgh, Sect. A 270, 103 (1962).

[3] A. Strominger, Lectures on the infrared structure of gravity and gauge theories, arXiv:1703.05448.

[4] G. Barnich and C. Troessaert, Aspects of the BMS/CFT correspondence, J. High Energy Phys. 05 (2010) 062.

[5] G. Barnich and C. Troessaert, Symmetries of Asymptotically Flat 4 Dimensional Spacetimes at Null Infinity Revisited, Phys. Rev. Lett. 105, 111103 (2010).

[6] G. Barnich and C. Troessaert, BMS charge algebra, J. High Energy Phys. 12 (2011) 105.

[7] E. E. Flanagan and D. A. Nichols, Conserved charges of the extended Bondi-Metzner-Sachs algebra, Phys. Rev. D 95, 044002 (2017).

[8] G. Compere and J. Long, Vacua of the gravitational field, J. High Energy Phys. 07 (2016) 137.

[9] G. Compere and J. Long, Classical static final state of collapse with supertranslation memory, Classical Quantum Gravity 33, 195001 (2016).

[10] P. P. Kulish and L. D. Faddeev, Asymptotic conditions and infrared divergencies in quantum electrodynamics, Theor. Math. Phys. 4, 745 (1971).

[11] D. Carney, L. Chaurette, D. Neuenfeld, and G.Semenoff, Infrared Quantum Information, Phys. Rev. Lett. 119, 180502 (2017).

[12] A. Strominger, Black hole information revisited, arXiv: 1706.07143.

[13] B. Carter, Axisymmetric Black Hole has Only Two Degrees of Freedom, Phys. Rev. Lett. 26, 331 (1971).

[14] D. C. Robinson, Uniqueness of the Kerr Black Hole, Phys. Rev. Lett. 34, 905 (1975).

[15] S. W. Hawking, M. J. Perry, and A. Strominger, Soft Hair on Black Holes, Phys. Rev. Lett. 116, 231301 (2016).
[16] S. W. Hawking, M. J. Perry, and A. Strominger, Superrotation charge and supertranslation hair on black holes, J. High Energy Phys. 05 (2017) 161.

[17] R. Bousso and M. Porrati, Soft hair as a soft wig, Classical Quantum Gravity 34, 204001 (2017).

[18] R. Bousso and M. Porrati, Observable supertranslations, Phys. Rev. D 96, 103512 (2017).

[19] I. Booth, Spacetime near isolated and dynamical trapping horizons, Phys. Rev. D 87, 024008 (2013).

[20] H. Friedrich, I. Racz, and R. M. Wald, On the rigidity theorem for spacetimes with a stationary event horizon or a compact Cauchy horizon, Commun. Math. Phys. 204, 691 (1999).

[21] S. Carlip, Entropy from conformal field theory at Killing horizons, Classical Quantum Gravity 16, 3327 (1999).

[22] J.-i. Koga, Asymptotic symmetries on Killing horizons, Phys. Rev. D 64, 124012 (2001).

[23] M. Hotta, K. Sasaki, and T. Sasaki, Diffeomorphism on horizon as an asymptotic isometry of Schwarzschild black hole, Classical Quantum Gravity 18, 1823 (2001).

[24] B. R. Majhi and T. Padmanabhan, Noether Current, horizon Virasoro algebra and entropy, Phys. Rev. D 85, 084040 (2012).

[25] L. Donnay, G. Giribet, H. A. Gonzalez, and M. Pino, Supertranslations and Superrotations at the Black Hole Horizon, Phys. Rev. Lett. 116, 091101 (2016).

[26] L. Donnay, G. Giribet, H. A. Gonzalez, and M. Pino, Extended symmetries at the black hole horizon, J. High Energy Phys. 09 (2016) 100.

[27] E. T. Akhmedov and M. Godazgar, Symmetries at the black hole horizon, Phys. Rev. D 96, 104025 (2017).

[28] M. R. Setare and H. Adami, BMS type symmetries at nullinfinity and near horizon of non-extermal black holes, Eur. Phys. J. C 76, 687 (2016).

[29] M.Z. Iofa, Thermal Hawking radiation of black hole with supertranslation field, J. High Energy Phys. 01 (2018) 137. 
[30] L. D. Landau and E. M. Lifschiz, Classical Theory of Fields (Moscow, Nauka, 1990).

[31] G. Barnich and F. Brandt, Covariant theory of asymptotic symmetries, conservation laws and central charges, Nucl. Phys. B633, 3 (2002).
[32] G. Barnich and G. Compere, Surface charge algebra in gauge theories and thermodynamic integrability, J. Math. Phys. (N.Y.) 49, 042901 (2008).

[33] S. Chandrasekhar, The Mathematical Theory of Black Holes (Clarendon Press Oxford, Oxford University Press, 1983). 